\title{
Tablet Triturate Dosage Form
}

National Cancer Institute

\section{Source}

National Cancer Institute. Tablet Triturate Dosage Form. NCI Thesaurus. Code C42759.

A compressed cylindrical tablet composed of medicated powder and dispersed with a mixture of lactose, powdered sucrose and a moistening agent. 